\title{
Leaders
}

\section{Mycological techniques}

\author{
K G Davey, C K Campbell, D W Warnock
}

\begin{abstract}
Introduction
Laboratory tests are essential to establish the diagnosis of a fungal infection. The methods used are based on three broad approaches: (1) the detection of the pathogen in tissue on direct microscopic examination; (2) its isolation and identification in culture; and (3) the detection of an immunological response to the pathogen. This Broadsheet describes the collection of clinical specimens from patients with suspected fungal infection and outlines appropriate test procedures for the detection, isolation and identification of fungal pathogens. It does not cover the serological diagnosis of fungal infection.
\end{abstract}

\section{Collection of specimens}

It is important to provide the laboratory with adequate specimens for investigation together with sufficient clinical information about underlying illness, recent travel or previous residence abroad, animal contacts, and the patient's occupation if considered relevant. With the exception of skin, nails and hair, all samples should be collected in sterile containers.

\section{SKIN, NAILS AND HAIR}

Skin, nails and hair should be collected into folded squares of stiff black paper (about $10 \times 10 \mathrm{~cm}$ ). These can now be purchased from a number of commercial suppliers (Dermaco, PO Box 470, Toddington, Bedfordshire LU5 6BK; MycoTrans, PO Box 1172, Biggar, Lanarkshire ML12 $6 \mathrm{NN}$ ). It is essential to clean superficial lesions with $70 \%$ alcohol prior to sampling if ointments, creams or powders have been applied. If pityriasis versicolor is a possible diagnosis, the affected sites should be examined under ultraviolet light (Wood's lamp). The characteristic golden yellow fluorescence often associated with this infection can help in the selection of skin scrapings.

Material should be collected from cutaneous lesions by scraping outwards from the margin of the lesion with a blunt scalpel. If there is minimal scaling, clear adhesive tape such as sellotape can be used to remove material for examination. The sellotape strip is pressed against the lesion, peeled off and placed, adhesive side down, on a clean glass microscope slide for transportation to the laboratory.

Nail scrapings or clippings should be taken from discoloured or dystrophic parts of the nail. Specimens should be cut as far back as possible from the free edge of the nail and should include its full thickness. Specimens should be taken from several nails if more than one is affected. Debris beneath infected nails often contains the fungus.

It is sometimes helpful to use a Wood's lamp to select infected scalp hairs for investigation. If none of the hairs gives the green fluorescence which is characteristic of some microsporum infections, a search should be made for lustreless hairs or stumps and for hairs broken off at follicle mouths. Hairs should be extracted from the scalp using flat-ended forceps. Cut hairs without roots are unsuitable for mycological examination. Skin scrapings should also be collected from sites where fungal infection of hairs is suspected.

Another method which is useful for the collection of adequate material from patients with suspected scalp infection not detectable under a Wood's lamp is to brush the scalp with a plastic massage pad (available from most chemists), which is then pressed into the surface of an agar plate. The pad should be sterilised in $1 \%$ chlorhexidine for one hour, rinsed in sterile water and dried before being reused.

\section{MUCOUS MEMBRANES}

Swabs moistened in sterile water or saline should be used to collect exudate or discharge. The swabs should be sent to the laboratory in a standard transport medium.

\section{EAR}

Scrapings of material from the ear canal are to be preferred, although swabs can also be used.

\section{EYE}

Material from a corneal ulcer with a suspected fungal cause should be scraped with a sterile platinum spatula, sampling both the base of the ulcer and the edges. This procedure should be performed by an eye specialist. Because the amount of material that can be obtained will be small, it is best transferred to a glass slide for microscopic examination and to an agar plate for culture at the bedside. The culture plate should be marked to indicate the point of inoculation. Swabs are not suitable for sampling corneal lesions.

BLOOD

Blood culture is an important part of the investigation of suspected deep fungal infection. 
However, microbiologists should not expect blood cultures taken for bacterial isolation to detect fungal pathogens other than Candida spp., Cryptococcus neoformans, or Trichosporon spp. Isolation of fungi from blood depends on a number of factors including the amount of blood sampled and the method of processing. Commercial biphasic and Bactec blood culture systems will often permit the detection of fungal infection.

SPUTUM AND BRONCHOALVEOLAR LAVAGE

Fresh early morning samples of sputum are ideal. These should be collected in sterile containers and processed within two hours of collection. If delay in processing is unavoidable, specimens should be stored at $4^{\circ} \mathrm{C}$. More invasive sampling methods are often required to obtain specimens from neutropenic patients. Broncheoalveolar lavage (BAL) provides useful samples for direct microscopic examination and culture.

\section{CEREBROSPINAL FLUID, URINE AND OTHER}

FLUIDS

Cerebrospinal fluid samples of 3-5 $\mathrm{ml}$ are ideal, but are usually smaller than this. Samples can be centrifuged and the supernatant fluid used for serological tests. The sediment can be cultured, but is also useful for direct microscopic examination. In non-catheterised patients, fresh mid-stream specimens of urine are adequate for mycological investigation, provided care is taken to ensure that vaginal or perineal infection does not lead to contamination. In infants, suprapubic aspiration is the best method of urine collection. Other fluids, whether aspirated or drained, should be collected into sterile containers which include a small amount of sterile heparin diluted 1 in 1000 to prevent clotting. These specimens should be centrifuged and the sediment cultured. Drain fluid from patients receiving continuous peritoneal dialysis should be collected into sterile containers without heparin.

\section{PUS, BONE MARROW AND TISSUE}

If possible, swabs should not be used to collect material from draining abscesses or ulcers. If a swab must be used, then material should be taken from as deep as possible within the lesion. Pus from undrained subcutaneous abscesses or sinus tracts should be collected with a sterile needle and syringe. If grains are visible in the pus (as in mycetoma), these must be collected. Bone marrow specimens should be collected into sterile containers which include a small amount of sterile heparin diluted 1 in 1000 . Tissue specimens should be placed in sterile saline. If possible, material should be obtained from both the middle and the edge of lesions.

\section{Processing of specimens}

SKIN, NAILS AND HAIR

Skin and nail specimens should be chopped into small fragments. If, as is usual, the speci-
Characteristics of some common dermatophytes invading hair

\begin{tabular}{lll}
\hline Fungus & $\begin{array}{l}\text { Arthrospore size } \\
(\mu \mathrm{m})\end{array}$ & Arrangement \\
\hline Microsporum audouinii & Small $(2-5)$ & Ectothrix \\
Microsporum canis & Small (2-5) & Ectothrix \\
Trichophyton mentagrophytes & Small (3-5) & Ectothrix \\
Trichophyton tonsurans & Large (4-8) & Endothrix \\
Trichophyton violaceum & Large (4-8) & Endothrix \\
Trichophyton verrucosum & Large (5-10) & Ectothrix \\
\hline
\end{tabular}

men consists of more than one piece of material, it is advisable to use some of each for microscopic examination and culture. Hairs should first be examined under a Wood's lamp and fluorescent parts, if present, used for processing. Hairs should be cut about $5 \mathrm{~mm}$ above the root and the upper portion discarded. Selected fragments or roots are placed in a large drop of $20 \%$ potassium hydroxide $(\mathrm{KOH})$ on a microscope slide and a coverslip placed on top. The slide should be set to one side to soften and clear the material. Skin material will clear in 15 to 20 minutes, as will thin nail parings and sub-ungual debris, but thicker nail fragments can take 30 to 60 minutes to soften. Gentle warming of preparations will reduce the time required for clearing, but care must be taken to avoid boiling.

Once skin or nail material has digested, the coverslip should be pressed down to squash out the fragments and render them transparent, blotting off the excess $\mathrm{KOH}$. Hair specimens should not be heated or squashed as infected hairs will disintegrate and the diagnostic arrangement of the arthrospores will be lost.

Low power magnification ( $x 10$ objective) is adequate for the detection of fungal hyphae in $\mathrm{KOH}$ preparations, but a higher power $(\times 40$ objective) is often required to confirm their presence. Septate hyphae which may consist of chains of rectangular spores (termed arthrospores) are typical of dermatophyte infection. In cases of pityriasis versicolor, the fungus (Malassezia furfur) appears as clusters of round cells together with short, unbranched hyphae. If a hair specimen is found to be infected, the size of the arthrospores and their arrangement should be noted (table).

Care must be taken to distinguish fungal hyphae from the artefact known as "mosaic". This is thought to be caused by deposition of cholesterol and, unlike true fungus, it tends to follow the outlines of the epidermal cell walls. Methods for selective staining of fungal hyphae are available, but are seldom required.

The recognition of fungal hyphae and/or arthrospores by direct microscopic examination is sufficient for the diagnosis of a dermatophyte infection, but gives no indication of the species of dermatophyte involved. Isolation should be attempted whether or not fungus has been found on microscopic examination. If there is insufficient material for both microscopic examination and culture of a specimen, the former should be performed, unless the clinician has done it. It is important to remember that while fungus found on microscopic ex- 
amination may fail to grow in culture, the opposite is also true.

Isolation is best made on agar plates. Skin, nail and hair specimens should be cultured on Sabouraud's dextrose agar (glucose peptone agar) supplemented with chloramphenicol $(50 \mathrm{mg} / \mathrm{l})$ to reduce bacterial contamination and cycloheximide (actidione, $50 \mathrm{mg} / \mathrm{l}$ ) to suppress most non-dermatophyte fungi. Malt agar can be used in place of Sabouraud's dextrose agar. Up to 20 skin or nail fragments, or hair roots should be pressed into the surface of a culture plate. If skin or nail material is suspected of being infected with moulds, such as Scytalidium spp. or Scopulariopsis brevicaulis, it is important to set up duplicate plates of Sabouraud's dextrose agar with and without cycloheximide. If sellotape strips are received, these are detached from the microscope slides and placed on the surface of a Sabouraud's dextrose agar plate. Incubation at room temperature is adequate for the isolation of dermatophytes, but $27-30^{\circ} \mathrm{C}$ is preferred.

Sabouraud's dextrose agar can be obtained from a number of commercial suppliers, but as the morphological appearance of the fungi, and pigmentation in particular, often differs from one medium to another, it is advisable to confine supplies to one manufacturer. This also applies to the nature of the peptone if the medium is prepared in house.

Most dermatophyte cultures can be identified after seven to 10 days of incubation at $30^{\circ} \mathrm{C}$ (see later). If there is no growth after this time, the plate can be discarded as negative. If growth is apparent, the plate should be reincubated until the colonies are large enough to be identified. If there is no growth from material which was positive on microscopic examination and further material is available, it is advisable to set up a further culture.

Moulds other than dermatophytes are sometimes recovered from abnormal skin and nails. In most cases, these are casual, transient contaminants and direct microscopic examination of scrapings is negative. However, certain moulds are capable of causing infection and when this is so, it is important that their significance is recognised. Scytalidium dimidiatum (Hendersonula toruloidea) and Scytalidium hyalinum have been isolated from hand, foot and nail infections. Microscopic examination of infected material shows chains of hyaline arthrospores which may be difficult to distinguish from those of a dermatophyte. These fungi are sensitive to cycloheximide and this antibiotic should be omitted from the culture medium. $S$ brevicaulis is the most common non-dermatophyte cause of nail infection. It is possible to distinguish infections with this mould on direct microscopic examination of nail specimens if the characteristic roughened oval spores are found.

\section{OTHER SPECIMENS}

Sputum and BAL specimens should be regarded as hazardous, due to potential mycobacterial infection, and should be processed inside a class 1 safety cabinet in a containment level 3 laboratory. Tissues, fluids and pus need not be processed inside a cabinet. However, if a mould is isolated and the information provided with the specimen suggests a diagnosis of blastomycosis, coccidioidomycosis, histoplasmosis, paracoccidioidomycosis, or penicilliosis, all further work must be performed inside a cabinet.

Swabs should be cultured on Sabouraud's dextrose or malt agar supplemented with chloramphenicol. Plates should be incubated at $37^{\circ} \mathrm{C}$ for up to one week and examined at 48 hour intervals. Swaiss should be spread over the surface of a culture plate, then dipped in a drop of $20 \% \mathrm{KOH}$ on a clean slide to wash off material for direct microscopic examination.

Sputum should be digested prior to microscopic examination and culture. An amount of Sputasol (or similar reagent), equal to that of the sputum, is added to the specimen container. The specimen is incubated at $37^{\circ} \mathrm{C}$ for 30 minutes, then centrifuged and a portion of the sediment spread over the surface of a Sabouraud's dextrose or malt agar plate (supplemented with chloramphenicol). This should be incubated at $37^{\circ} \mathrm{C}$ for 48 to 72 hours. For direct microscopic examination, a portion of sediment is added to a drop of $20 \% \mathrm{KOH}$ on a clean slide and a coverslip added.

In general, blood cultures should be incubated for seven to 10 days. Those showing apparent growth should be subjected to direct microscopic examination. If yeast cells are present, an Indian ink preparation should be made to demonstrate the possible presence of encapsulated $C$ neoformans cells (see below). Irrespective of apparent growth, all blood cultures should be sub-cultured on to $\mathrm{Sa}$ bouraud's dextrose agar plates for incubation at 30 and $37^{\circ} \mathrm{C}$ for two weeks. If the clinical details suggest infection with a Hazard Group 3 pathogen (see below), screw-capped sloped containers should be used instead of plates.

Fluids should be centrifuged and the deposit used for microscopic examination and culture. If cerebrospinal fluid samples are being examined, an Indian ink preparation should be made to demonstrate the possible presence of encapsulated $C$ neoformans cells (see later).

Tissue samples should be placed in a sterile Petri dish and chopped into small fragments prior to culture. Selected fragments should be placed in a large drop of $20 \% \mathrm{KOH}$ on a microscope slide and a coverslip placed on top. The slide should be set to one side to soften and clear the material. For culture, up to 20 fragments are pressed into the surface of Sabouraud's dextrose or malt agar plates (supplemented with chloramphenicol). These should be incubated at 30 and $37^{\circ} \mathrm{C}$ for up to two weeks and examined at 48 hour intervals.

If the clinical information provided with a specimen suggests a diagnosis of blastomycosis, coccidioidomycosis, histoplasmosis, paracoccidioidomycosis, or penicilliosis, some modification of culture methods is advisable. For safety, such specimens should be cultured in screw-capped sloped containers rather than on plates. Duplicate cultures should be set up on Sabouraud's dextrose or malt agar (supplemented with chloramphenicol) at $30^{\circ} \mathrm{C}$ and 
blood agar or brain heart infusion agar at $37^{\circ} \mathrm{C}$. The slopes should be incubated for three to four weeks before being discarded as negative.

\section{Identification methods}

MOULDS AND DERMATOPHYTES

Moulds and dermatophytes are identified on the basis of their colonial and microscopic morphological characteristics. Macroscopic features, such as colonial form, surface colour and the production of pigments, are helpful in identification: moulds with blue, green or black colonies are not dermatophytes. In some cases, for instance Trichophyton verrucosum and Trichophyton soudanense, the diagnostic characteristics are most easily seen by examining the reverse side of the plate under the low power objective of a microscope.

\section{Needle mount}

This is the most important part of the examination of a culture. If well prepared, a needle mount will often give sufficient information on the form and arrangement of spores and other structures for identification of the fungus to be made. Using a sterilised rigid sharp needle, some of the surface growth is removed from a culture plate and placed in a drop of lactophenol cotton blue stain on a clean microscope slide. The material is then teased apart with two sharp needles and a coverslip is applied. Gentle pressure is used to spread out the preparation before it is examined under a microscope using the $\times 10$ and $\times 40$ objectives. If no spores are found in slide mounts, it is sometimes worthwhile to remove the lid from the culture plate and examine the colonies for evidence of sporulation under the low power objective of a microscope.

\section{Sellotape mounts}

These are useful for examining sporulating cultures. Using sterilised forceps, a small piece of sellotape, adhesive side down, is pressed on to the surface of the culture. The coated sellotape is then placed, adhesive side up, on to a drop of lactophenol cotton blue on a microscope slide. A further drop of lactophenol cotton blue is placed on the preparation and a coverslip applied.

\section{Slide culture}

The slide culture technique is useful for observing the intact arrangement of spores or spore bearing structures. A thin square block of agar is placed on a sterile microscope slide supported on a bent glass rod in a Petri dish. The four sides of the agar block are then inoculated with portions of mycelium of the fungus to be identified. The block is then covered with a sterile coverslip, sterile distilled water is added to the base of the Petri dish, the lid is replaced and the plate is incubated at $30^{\circ} \mathrm{C}$. Once adequate sporulation has occurred the cover slip is removed from the agar and placed to one side with the adherent mycelium uppermost. The agar block is then removed and discarded, leaving adherent mycelium on the slide. Lactophenol cotton blue is added and a clean coverslip applied. The preparation can be sealed for long term preservation.

\section{Special media}

A medium which will assist the non-specialist to distinguish dermatophytes from other moulds is Dermatophyte Test Medium. A change in the amber colour of the phenol red indicator incorporated in the medium to pink or red denotes a change in $\mathrm{pH}$ and permits the presumptive identification of the isolate as a dermatophyte. It is important to remember that some non-dermatophyte moulds can also produce this colour change.

The urease test can often be used to distinguish Trichophyton rubrum isolates. A small portion of growth is removed from a culture plate with a rigid needle and used to inoculate a Christensen's urea slope. This is incubated at $30^{\circ} \mathrm{C}$ for one week. If the colour of the medium changes to red, the test is positive and the isolate is not $T$ rubrum. However, it is important to note that the granular form of $T$ rubrum, like most other dermatophytes, gives positive results.

\section{YEASTS}

A number of simple rapid tests have been devised for the identification of some of the most important yeast fungi. These include the serum germ tube test for Candida albicans and the urease test for $C$ neoformans. However, the first step is to note the colour and appearance of the colonies. Pink or coral colonies are suggestive of Rhodotorula spp. while cream or white colonies are suggestive of Candida spp., Cryptococcus spp., Trichosporon spp., or Geotrichum spp. Brown or black colonies are suggestive of Exophiala spp.

\section{Indian ink preparation}

If the information provided with the specimen suggests the culture is $C$ neoformans, an Indian ink preparation should be set up. $C$ neoformans produces round to oval or lemon-shaped cells with polysaccharide capsules. These capsules can be detected when the cells are mounted in a pigmented colloidal mounting fluid, such as Indian ink, which does not penetrate the capsular envelope. A light loopful of inoculum is taken from the culture and suspended in a drop of $50 \%$ aqueous Indian ink on a microscope slide. If a capsule is present, it should be visible as a clear halo around the cells. The presence of a capsule gives a presumptive identification of $C$ neoformans, but does not provide a definitive identification because other yeasts may be encapsulated. Moreover, not all $C$ neoformans isolates have prominent capsules and the capsule is often lost following subculture of the organism. 
Urease test

Most $C$ neoformans isolates possess the enzyme urease. A light loopful of inoculum is taken from the original isolation plate and spread over the surface of a Christensen's urea slope, which is then incubated at $30^{\circ} \mathrm{C}$ for up to four days. A colour change from amber to pink permits the presumptive identification of the isolate as $C$ neoformans. However, Trichosporon spp. can give a positive result and bacterial contamination can also result in a change in the colour of the medium.

\section{Germ tube test}

The formation of germ tubes in serum is a characteristic of most clinical isolates of $C$ albicans. A light loopful of inoculum is taken from a culture plate and suspended in $0.5 \mathrm{ml}$ of horse serum. This is incubated at $37^{\circ} \mathrm{C}$ for two to three hours. A drop of the suspension is then placed on a microscope slide, a coverslip is placed on top and the preparation examined under the microscope. In many instances of a positive test, fewer than $10 \%$ of yeast cells will produce germ tubes.

It is important to remember that about $5 \%$ of $C$ albicans isolates fail to produce germ tubes. Moreover, over-inoculation of the serum can result in inhibition of germ tube formation and too short an incubation period can lead to false negative results: two hours is a minimum not a maximum incubation time. Pseudohypha production can be mistaken for germ tube production. This can be recognised by a constriction at the junction between the mother cell and the false germ tube.

\section{Cornmeal agar culture}

Examination of the morphological characteristics of yeast isolates under the microscope is essential if species with identical physiological profiles are to be distinguished. Growth in microaerophilic conditions on cornmeal or other starch-containing media, such as rice agar, stimulates the formation of mycelium, pseudomycelium, arthrospores, and chlamydospores in those species able to produce them.

Using a loop, touch five colonies on a plate and mix them on the agar surface. Use this to make a streak inoculation on the surface of a cornmeal agar plate. The centre of the streak is then covered with a sterile coverslip and the plate incubated for at least 48 hours at $30^{\circ} \mathrm{C}$. The lid is then removed and the plate examined under a microscope for true mycelium, pseudo- mycelium, arthrospores, and chlamydospores.

Chlamydospores (which can take up to 96 hours to develop) are indicative of $C$ albicans. Addition of $1 \%$ Tween 80 to the cornmeal agar stimulates their production. If arthrospores are present, the isolate is a presumptive Trichosporon $\mathrm{sp}$. If only pseudomycelium is found, the isolate is a Candida sp. other than $C$ albicans. It should be noted that Candida guilliermondii needs 72 to 96 hours of incubation to produce pseudomycelium while Trichosporon beigelii needs a similar amount of time to produce arthrospores.

\section{Further tests}

For those yeasts which are germ tube negative, identification may be made using one of the commercial identification systems, such as API $20 \mathrm{C}$ (BioMerieux) or AUXACOLOR (Sanofi Diagnostics Pasteur). However, it is essential to examine the morphological characteristics of all yeast isolates to avoid errors in identification, whatever system is used. Yeasts which cannot be identified with commercial systems should be sent to a reference laboratory for identification using traditional assimilation and fermentation procedures.

\section{HAZARDOUS FUNGI}

Five dimorphic fungi are classified as hazard group 3 pathogens. These are Blastomyces dermatitidis, Coccidioides immitis, Histoplasma capsulatum, Paracoccidioides brasiliensis, and Penicillium marneffei. These organisms must be handled inside a class 1 cabinet in a containment level 3 laboratory and should be cultured in sloped containers. Although the yeast form of these pathogens presents a lower risk of infection, these organisms will revert to a mycelial form after several days of incubation at $30^{\circ} \mathrm{C}$. Plates suspected to contain these organisms must be sealed at once and removed to a containment level 3 laboratory. Such cultures are best sent to a specialist mycology laboratory for identification.

\section{Further reading}

Campbell CK, Johnson EM, Philpot CM, Warnock DW. Identification of pathogenic fungi. London: Public Health Laborat ory Service, 1996 (in press)

Evans EGV, Richardson MD (eds). Medical mycology: a practical approach. Oxford: IRL Press at Oxford University Press, 1989. Kwon-Chung KJ, Bennett JE. Medical mycology. 4th edn. Philadelphia: Lea and Febiger, 1992.

Richardson MD, Warnock DW. Fungal infection: diagnosis and management. Oxford: Blackwell Scientific Publications, 1993. Rippon JW. Medical mycology: the pathogenic fungi and the pathogenic actinomycetes. 3rd edn. Philadelphia: Saunders, 1988. 